\title{
Interprofessional approach to the management of functional neurological symptoms with co-morbid concussions and hypermobility syndrome: a case report
}

\author{
Marianna Kogut PT, DPT, CBIS, PCS* and Janice Laux, PT, MS, DOMP, PCS
}

Kennedy Krieger Institute, Baltimore, MD, USA

Abstract

Clinical education scenario: This case report describes the individualized treatment approach and clinical reasoning of a residency-trained physical therapist for an adolescent girl with symptoms of functional neurological symptom disorder (FNSD).

Rationale for case study: There are limited research studies regarding successful treatment of pediatric FNSD. This case study provides evidence for an interprofessional treatment approach of an adolescent with symptoms of FNSD, including the role of a residency-trained physical therapist.

Case description: A 15-year-old girl with symptoms of FNSD, multiple comorbidities, and history of concussions participated in an interprofessional treatment program lasting 1 year. Her treatment was assessed using the Energy Expenditure Index, Timed Up and Down Stairs, Five-Times Sit to Stand, 6-Minute Walk Test, and Functional Disability Index. The patient demonstrated improvement in all outcome measures post-treatment.

Implications: The patient's function was restored using an interprofessional treatment approach, which emphasized interpersonal communication. While future studies are needed to assess the efficacy of this treatment approach, this case report demonstrates that physical therapy residency training prepares a clinician to effectively treat complex patients with multi-system involvement.

MeSH terms: conversion disorder; physical therapy specialty

Keywords: Functional Neurological Syndrome Disorder (FNSD); Adolescent; Physical therapy residency; Interprofessional; Non-epileptic seizures (NES); Case report

Received: I I December 2020; Revised: I 4 July 2021; Accepted: 19 July 202 I; Published: 05 November 202 I

$\mathrm{F}$ unctional neurological symptom disorder (FNSD) is altered voluntary motor or sensory function incompatible with recognized neurological or medical conditions, and is theorized to be caused by psychological or psychosocial stressors. ${ }^{1}$ Children with FNSD are often 10-14 year-old girls who experience high demands in their academic, sporting, cultural, and social lives. ${ }^{2}$ The symptomatology may include motor weakness or paralysis; abnormal movements; non-epileptic seizures (NES); altered sensation; diminished consciousness; or loss of vision, speech, or hearing. ${ }^{3}$ The risk of acquiring
NES with FNSD increases if the child has sustained a mild head injury. ${ }^{2}$ Additionally, these children often present with one or more comorbidities, including anxiety, depression, and/or pain. ${ }^{1}$

Caring for adolescents with symptoms of FNSD requires exceptional skill in managing the complex, often variable, presentation over a prolonged course of treatment. Pediatric physical therapy (PT) residents (i.e. physical therapists participating in advanced training in a specialized area) are equipped to provide such care because of extensive mentorship and educational 
experiences in clinical reasoning, patient management, and communication. This desire to provide better patient care is a major motivator to pursue residency training. ${ }^{4}$

The purpose of this case report was to describe the successful interprofessional rehabilitation of an adolescent girl with FNSD symptoms, including the clinical reasoning and decision-making abilities of a pediatric PT resident involved in her care. The Johns Hopkins Medicine IRB does not require a protocol for case reports. ${ }^{5}$

\section{Clinical case scenario}

The patient, a 15-year-old student-athlete, with FNSD symptoms was referred to outpatient PT from an interprofessional pain management clinic. She presented with lower extremity pain; weakness; pseudosyncopal episodes (PSE); and decline in school, social, and athletic participation. She had a significant history of three concussions in 4 years. Behavioral psychology and/or PT successfully treated her first two concussions; however, her symptoms remained unresolved following her third concussion (Fig. 1). Her comorbidities included hypermobility syndrome, dysautonomia, and chronic pain syndrome. There was a significant family history of parental psychiatric and cardiac hospitalizations. She required skilled and highly collaborative interprofessional outpatient and inpatient rehabilitation for nearly 1 year to facilitate return to her prior functional level (Fig. 1).

The plan of care (POC) was organized into three consecutive phases, distinguished by clinical setting (Fig. 1). Phase 1 involved hospital-based outpatient medical PT, occupational therapy (OT), and behavioral psychology evaluations. The PT examination followed a systems-based approach to test the hypotheses of cranial/cervical/vestibular dysfunction versus FNSD. The patient's strength, sensation, balance, vestibular, ocular-motor, and endurance were examined, the findings of which were negative for vestibular or ocular-motor dysfunction, radicular neuropathy, and peripheral neuropathy; however, positive for joint hypermobility, generalized weakness, and decreased physical activity tolerance.

Residency training emphasizes the importance of connecting elements of the International Classification of Function (ICF) model to clinical decisions. The patient's ICF schema guided selection and administration of pre-rehabilitation standardized outcome measures to include impairment-based (Energy Expenditure Index), activity-based (Five-Times Sit to Stand, Timed Up and Down Stairs, and 6-Minute Walk Test), and participation-based (Functional Disability Index) outcome measures. ${ }^{6-10}$ Table 1 depicts results of Phase 1 testing.

Interprofessional communication between outpatient PT, OT, behavioral psychology and physicians confirmed variable symptomatology unexplained by somatic or psychological findings alone. Notably, the patient reported a high level of activity on the day of evaluation, discrepant from her medical record review (Table 1). Prior and concurrent medical, laboratory, and imaging diagnostic work-up ruled out neurological, vascular, neuromuscular, or cardiorespiratory etiologies for her symptoms. Following interprofessional communication, the patient was diagnosed with symptoms of FNSD and referred for inpatient rehabilitation. Several months of additional

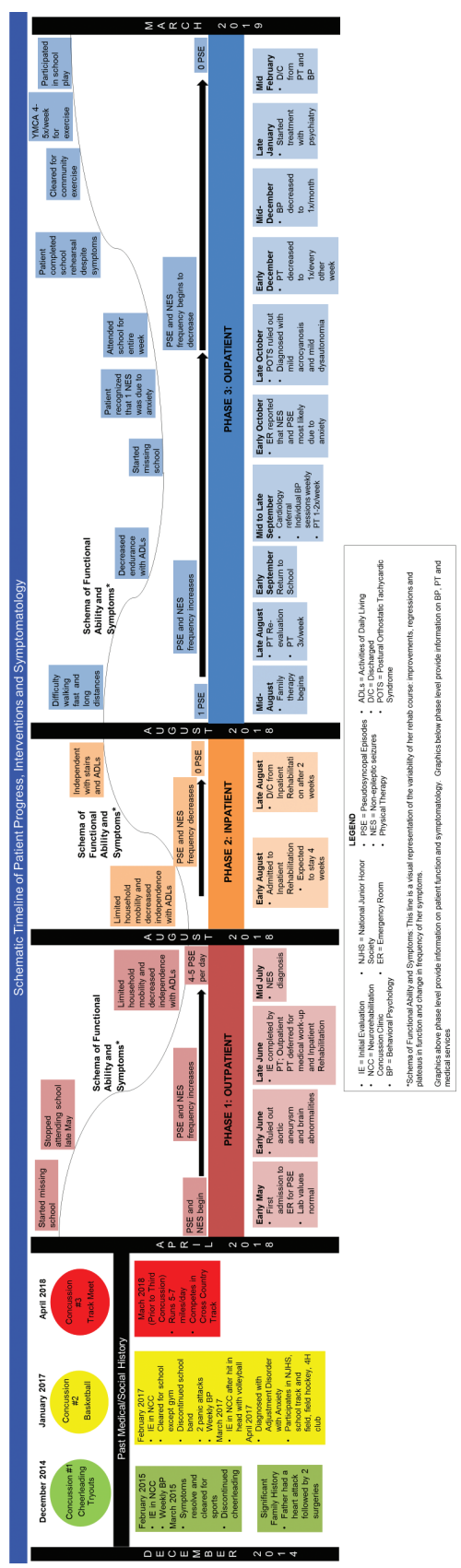

Fig. 1. Timeline of patient progress, interventions, and symptomatology.

*Enlarged image of timeline on Pages 4 and 5. 
Table 1. Outcome measures and patient perception

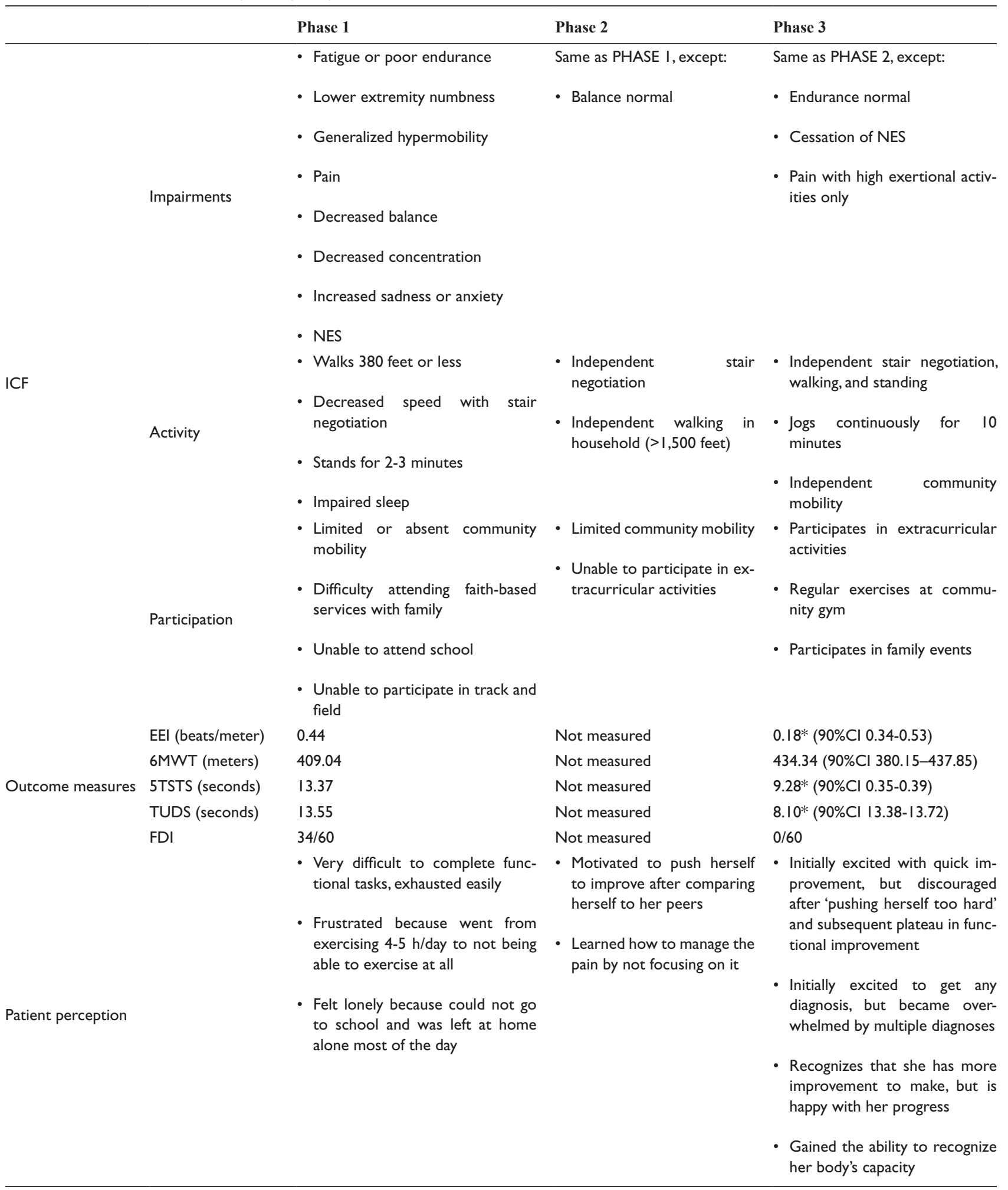

Abbreviations: ICF = International Classification of Functioning, Disability and Health model ICF; NES = Non-epileptic seizures; BHS = Beighton Hypermobility Scalew; EEI = Energy Expenditure Index; 6MWT = 6 Minute Walk Test; 5 TSTS = 5 times sit to stand;TUDS = Timed Up Down Stairs; FDI = Functional Disability Index; $\mathrm{Cl}=$ Confidence Interval.

*Indicates a statistically rare change. 


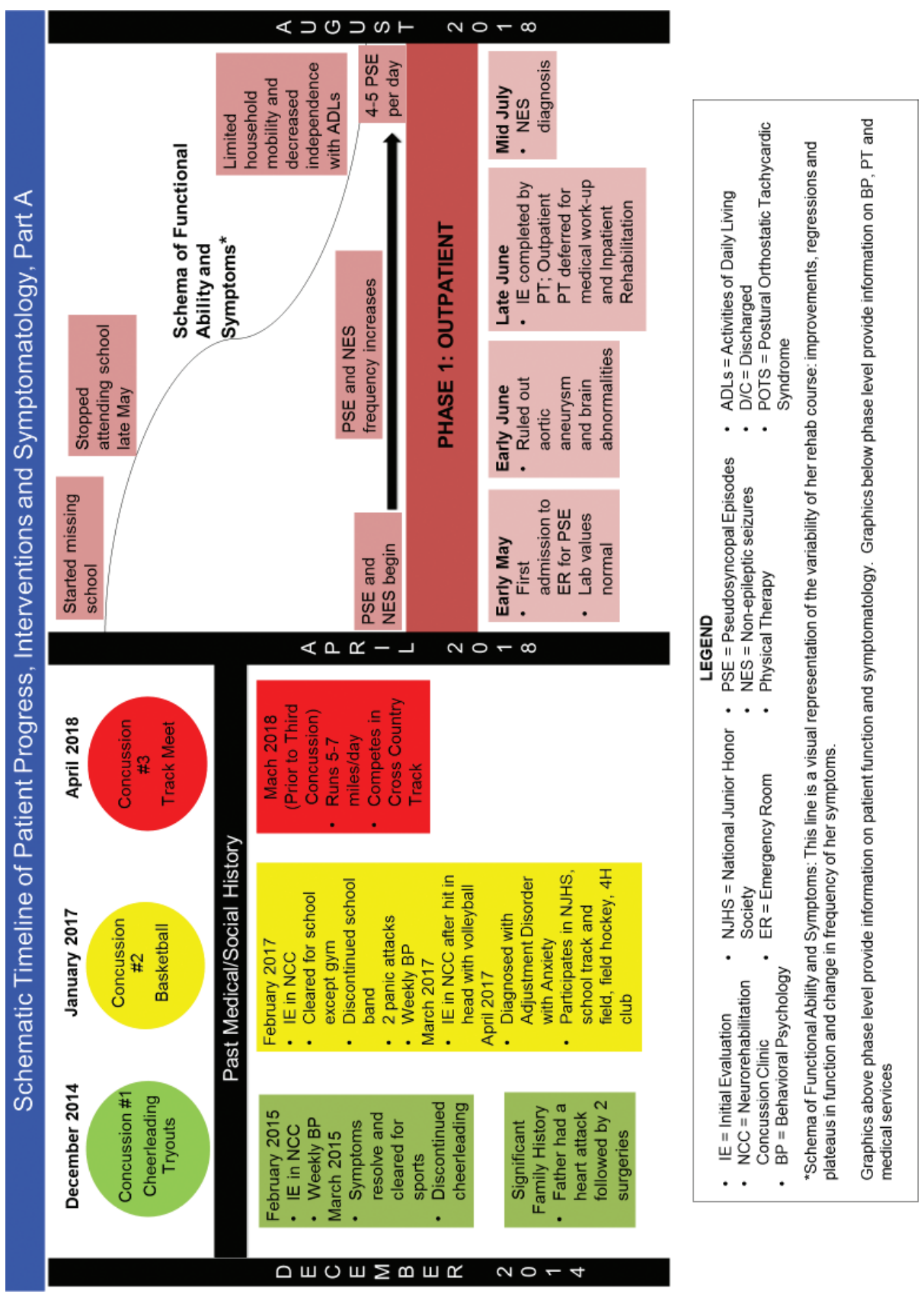

Fig. 1. Part A: Timeline of patient progress, interventions, and symptomatology.

medical work-up of her NES delayed outpatient PT and inpatient admission.

During Phase 2, the patient participated in 2 weeks of intensive inpatient rehabilitation. Care coordination between outpatient and inpatient PT occurred at the onset of inpatient admission. The patient's inpatient team included PT, OT, Child Life Services, behavioral psychology, and medical services. The inpatient PT POC included pain education, activity-based strengthening, endurance, and balance training. The pain neuroscience and rehabilitation literature emphasized focus be placed on function, not symptomatology. ${ }^{11-14}$ By inpatient discharge, the patient was independent in household ambulation and experienced no NES or PSE. Her functional progress, ability to use self-regulation strategies, and appropriate understanding of her pain justified her inpatient discharge. To continue progress toward functional independence and participation in school and community settings, outpatient PT services were recommended.

Outpatient treatment in Phase 3 focused on wellness, activity tolerance, and managing accessory motions (Fig. 2). The physical therapists resident became the primary 


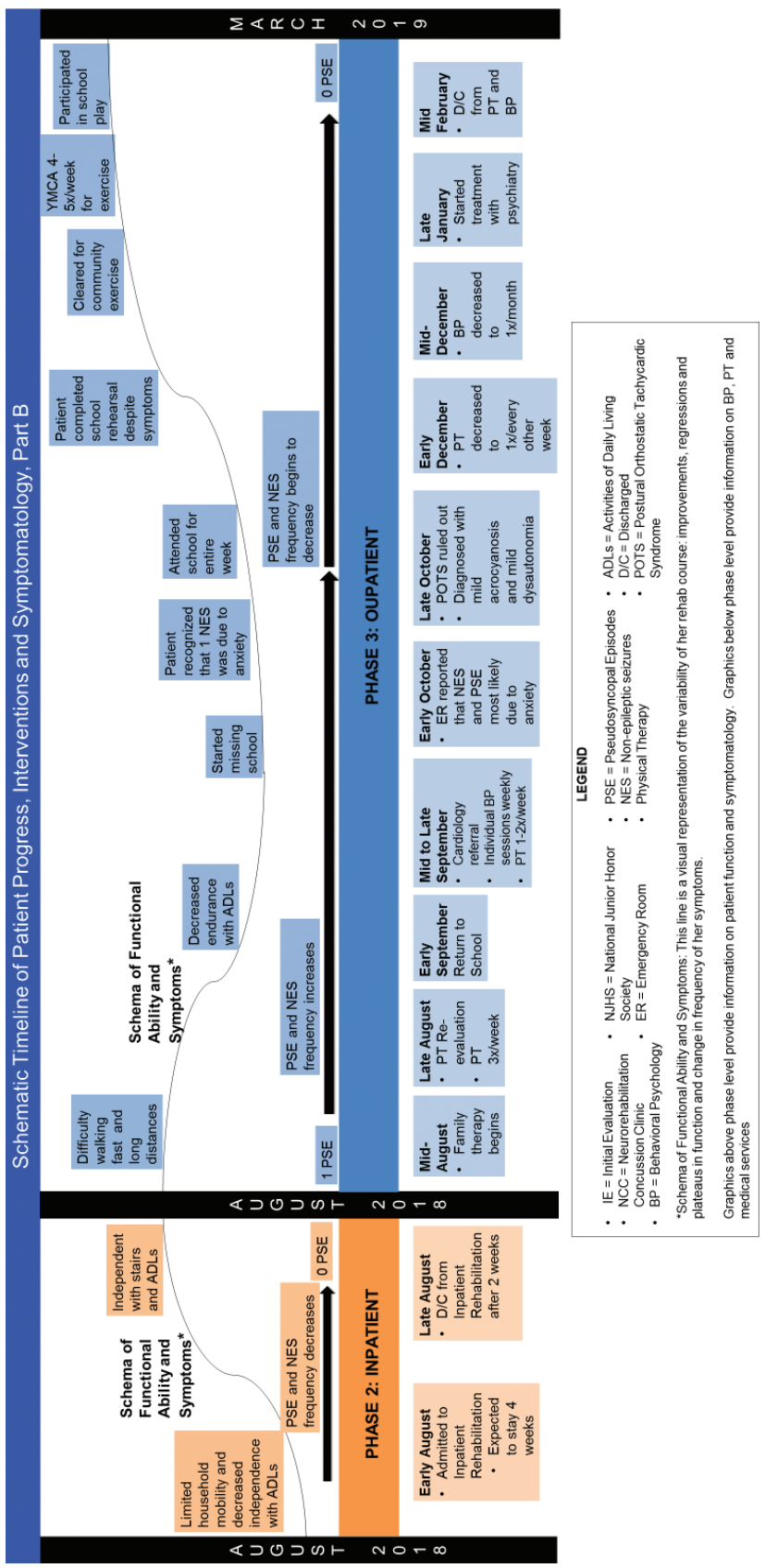

Fig. 1. Part B: Timeline of patient progress, interventions and symptomatology.

physical therapist during this phase. The patient's prior history and treatment, available evidence, and residency acquired appraisal skills, guided the resident in development of the outpatient POC. Available evidence indicated the use of joint stabilization techniques and regular paced physical activity to improve joint pain, increase strength, and build an endogenous locus of control to lessen anxiety. ${ }^{12-14}$ Intensity of services began with 60 -min sessions three times per week for 4 weeks, decreased to one to two times per week for the next 10 weeks, and further decreased to one to two times per month for the following 8 weeks before discharge from PT. This initial high intensity permitted close monitoring of the patient's physical activity, progress toward weekly goals, and updating her home program for optimal functional carryover. Therapeutic interventions were modified according to patient tolerance and progress toward functional weekly short-term objectives (Fig. 2). Patient tolerance to treatment was assessed through formal 30-day re-evaluations and each session using subjective report and informal behavioral assessment. Patient adherence was measured through progression of the individualized home program. 


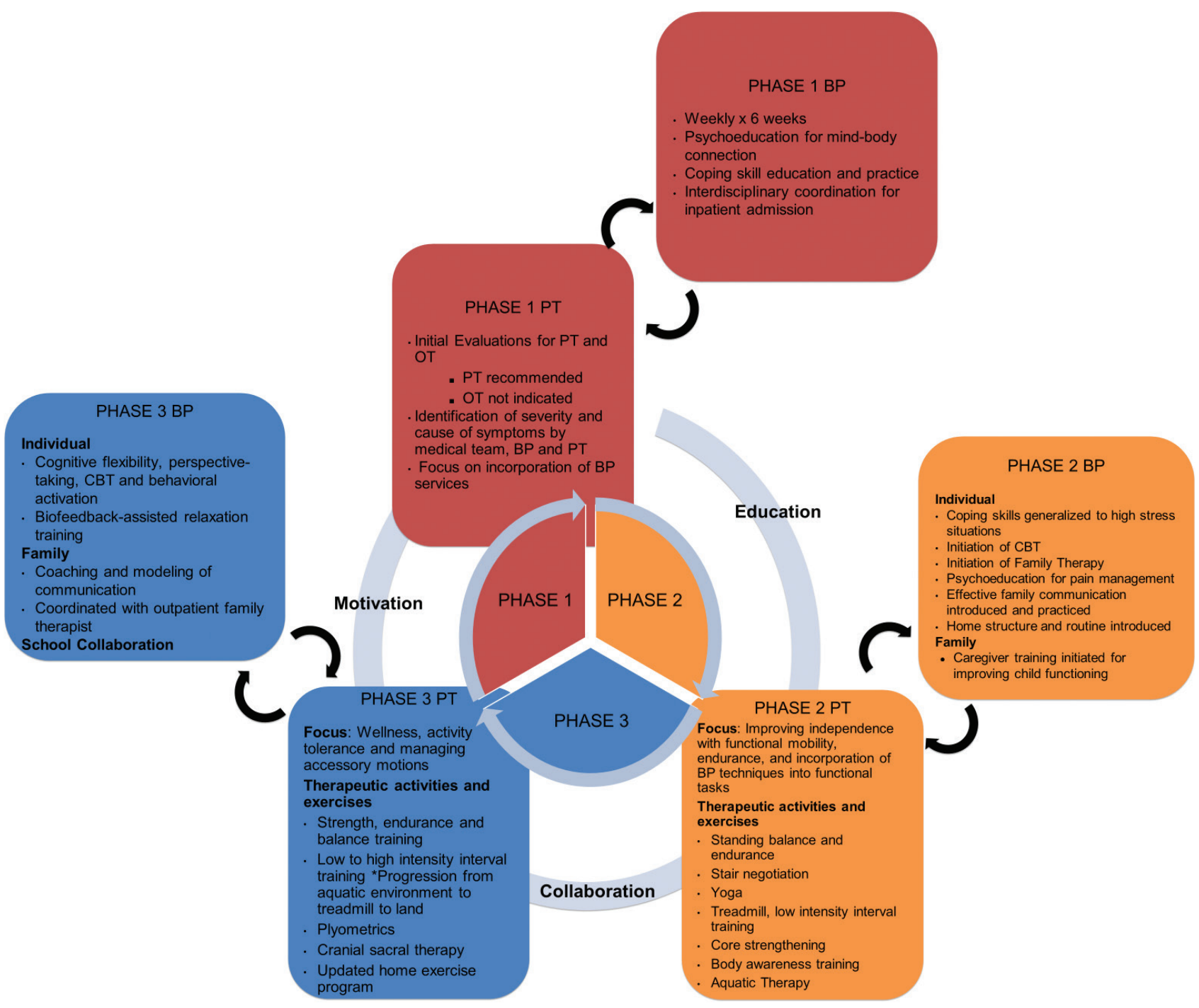

Fig. 2. Rehabilitation treatment program.

Abbreviations: $\mathrm{PT}=$ physical therapy; $\mathrm{BP}=$ behavioral psychology; $\mathrm{CBT}=$ cognitive behavioral therapy.

Figure 1 represents the variability in symptomatology and functional progress over the patient's treatment course. The patient experienced a return of NES and PSE during the beginning of Phase 3 (Fig. 1B). These symptoms coincided with return to school, decreasing her attendance. Four weeks later, her school attendance improved to $100 \%$, and she began to participate in new non-competitive extra-curricular activities. Neither NES nor PSE were reported in the last 12 weeks of Phase 3, and these symptoms remained absent 1-year post-initiation of treatment. The patient's symptoms and functional variability during Phase 3 were understood through skillful and compassionate communication between the resident and the patient. The resident fostered open communication through creation of a supportive and trusting environment. The education and mentorship the pediatric PT resident received from residency faculty senior physical therapists through didactics and clinical experience enhanced her ability to connect with this patient on a personal, yet professional level, and to acknowledge and validate the patient's perspective while still facilitating progress. After discharge from outpatient services, the patient attended school full time, participated in the spring musical and exercised at the gym 5 days per week with friends. Re-administration of all standardized outcome measures demonstrated improvement over baseline scores (Table 1). To evaluate whether changes in scores were statistically rare, the Reliable Change Index metric was used (Table 1). ${ }^{6-10}$ Statistically rare changes were found in Energy Expenditure Index, ${ }^{6,7}$ Five-Times Sit To Stand, ${ }^{6,9}$ and Timed Up and Down Stairs, ${ }^{10}$ indicating improvements in aerobic capacity, strength, speed, and efficiency of functional mobility (Table 1). Her 6-Minute Walk Test 
improved, but was not statistically rare (Table 1) ${ }^{6,8} \mathrm{Her}$ Functional Disability Index score decreased from 34/60 to $0 / 60$, indicating improvement in subjective perception of functional limitations. These changes demonstrated improvements in the impairment, activity and participation domains of the ICF and supported the decision for PT discharge.

During a motivational interview led by the PT resident 1 year post-onset of FNSD symptoms, the patient explained her perceptions of her PT treatment and outcomes (Table 1). Throughout all the three phases, the patient fluctuated between feeling excited, distressed, and overwhelmed. By discharge, she was pleased with her progress, with the recognition that she had more improvement to make. She had gained a new mindfulness, cognizance of her body's cues and capacity, and flexibility in redefining herself from an athlete to a performer. For the resident, this confirmed the importance of understanding and supporting the patient's perspective.

\section{Discussion}

While the exact mechanism for FNSD development is unknown, the recent literature demonstrates that there may be altered organization in the areas of the brain that modulate motor responses and emotional perception. ${ }^{1}$ More specifically, there may be over-activation in the prefrontal cortex and pre-motor or supplementary motor areas of the brain at rest. ${ }^{15}$ Therefore, children and adolescents with FNSD may have an experience-dependent plasticity pathway associated with increased vigilance to the emotions of others and increased motor readiness to organize self-protectively in the context of stressors. ${ }^{1}$ Treatment for individuals with FNSD often requires a lengthy interprofessional rehabilitation process across multiple settings, and may include individual and family psychotherapy, medication for comorbid anxiety and depression, PT, and OT. ${ }^{3}$ With complex patients, many clinicians look toward the literature for guidance. However, clinical guidance from research studies and the literature regarding treatment of these patients is sparse, especially in the pediatric literature.

The impact of FNSD on the patient is often profound. This patient declined from excelling as a student athlete to being homebound and isolated. The ambiguity inherent in FNSD symptomatology complicates conceptualization of the situation by everyone involved in the patient's care. FNSD often adversely affects patient and family relationships, and places strain on the entire family. Successful treatment is challenging, requiring a coordinated, interprofessional approach. This patient was predisposed to FNSD symptoms by her history of multiple concussions, pre-existing family stressors, and conflicting demands from different areas of her life. ${ }^{1}$ Her course was further complicated by her comorbidities of anxiety, hypermobility syndrome, and dysautonomia. After nearly 1 year of interprofessional treatment, the patient improved in all ICF model domains.

Standardized and validated outcome measures were important in this case report to compare pre- and post-treatment functional abilities. Reflection, through mentoring and the ICF model, guided the resident in linking the patient's impairments, activity, participation, and contextual factors to the established function-based outcome measures and determination of the patient's POC.

Skilled communication and collaboration between PT, behavioral psychologist, the patient, and family were essential to successfully manage this patient's complex presentation. The physical therapist benefited from incorporating behavioral psychology coping strategies into therapeutic interventions designed to advance functional mobility. Similarly, behavioral psychology, through communication with PT about the patient's behavior and symptomatology in a functional context, was guided in identifying coping strategies. Most importantly, none of the interventions would have been successful without the providers' ability to actively listen to the patient and consider the patient's perceptions about his or her body, symptoms, and function.

While there are growing research studies on FNSD in adults, the literature regarding diagnosis and treatment of FNSD in children and adolescents is limited. Given the paucity of literature and the emphasis that residency training places on applying evidence-based practice, the authors were motivated to publish this case report. This report supports the importance of an interprofessional and individualized approach in the successful management of these patients. Additionally, this case report highlights how the resident's educational and clinical experiences, combined with mentorship, contributed to her care of this complex patient. Through the residency program, the physical therapist gained advanced communication skills, partly, through the multitude of opportunities to communicate with a variety of providers and patients across various settings. These were important factors in navigating the patient's care across practice settings and throughout various transitions for return to her prior functional level.

Owing to the nature of this case report, cause-effect relationships between treatment interventions and outcomes cannot be determined, nor can the results be generalized to the general population of adolescents with FNSD. However, given the significant improvements in function with intervention, especially following a decline just prior to treatment, the authors are hopeful that the rehabilitation program contributed to functional improvements.

\section{Implications}

Restoring function and maintaining long-term mental health of adolescents with FNSD require an interprofessional 
approach, advanced communication skills, and clinical reasoning abilities of all treating providers. Residency-trained physical therapists are aptly suited to provide the necessary high-quality care described in this case report because of intensive training in their specialty areas with an unparalleled focus on clinical mentorship. Additionally, future studies are needed to assess the efficacy of interprofessional treatment in the management of FNSD in the pediatric population.

\section{Conflict of interest and funding}

The authors have not received any funding or benefits from industry or elsewhere to conduct this study.

\section{Disclosure}

This research article was presented as a poster at Combined Sections Meeting of the American Physical Therapy Conference in 2020. The title of the poster was 'Multidisciplinary Approach to the Management of Functional Neurological Symptoms with Co-morbid Concussions and Hypermobility Syndrome'.

\section{Ethical statement}

Patient provided written consent for publication of this manuscript. No IRB completed.

\section{References}

1. Kozlowska K, Griffiths KR, Foster SL, et al. Grey matter abnormalities in children and adolescents with functional neurologic symptom disorder. Neuroimage Clin (2017) 15: 306-14. doi: 10.1016/j.nicl.2017.04.028

2. Lortie A. Psychogenic nonepileptic seizures. Handb Clin Neurol (2013) 112: 875-9. doi: 10.1016/B978-0-444-52910-7.00009-X

3. Grattan-Smith PJ, Dale RC. Pediatric functional neurologic symptoms. Handb Clin Neurol (2016)139: 489-98. doi: 10.1016/ B978-0-12-801772-2.00040-0

4. Osborne R, Janson C, Black L, et al. Motivations to pursue physical therapy residency training: a Q-methodology study of stakeholder perspectives. Phys Ther (2020) 100: 57-72. doi: 10.1093/ptj/pzz142

5. Internet document: Johns Hopkins Medicine. Organization policy on single case reports and case series. Available from: https://www.hopkinsmedicine.org/institutional_review_board/ guidelines_policies/organization_policies/102_3.html [cited 8 December 2020].

6. Jacobson NS, Truax P. Clinical significance: a statistical approach to defining meaningful change in psychotherapy research. J Consult Clin Psychol (1991) 59(1): 12-19. doi: 10.1037/0022-006X.59.1.12

7. Gupta S, Raja K. Energy expenditure index as a measure of efficiency of walking on outdoor uneven surface in individuals with cerebral palsy. Disabil Rehabil (2019) 22: 1-8. doi: 10.1080/09638288.2019.1630017

8. Li AM, Yin J, Yu CC, et al. The six-minute walk test in healthy children: reliability and validity. Eur Respir J (2005) 25(6): 1057 60. doi: 10.1183/09031936.05.00134904

9. Wang T, Liao H, Peng Y. Reliability and validity of the five-repetition sit-to-stand test for children with cerebral palsy. Clin Rehabil (2011) 26(7): 664-71. doi: 10.1177/0269215511426889

10. Zaino CA, Marchese VG, Westcott SL. Timed up and down stairs test: preliminary reliability and validity of a new measure of functional mobility. Pediatr Phys Ther (2004) 16(2): 90-8. doi: 10.1097/01.PEP.0000127564.08922.6A

11. Cox T, Louw A, Puentedura EJ. An abbreviated therapeutic neuroscience education session improves pain knowledge in first-year physical therapy students but does not change attitudes or beliefs. J Man Manip Ther (2017) 25(1): 11-21. doi: 10.1080/10669817.2015.1122308

12. Maynard CS, Amari A, Wieczorek B, et al. Interdisciplinary behavioral rehabilitation of pediatric pain-associated disability: retrospective review of an inpatient treatment protocol. J Pediatr Psychol (2010) 35: 128-37. doi: 10.1093/jpepsy/jsp038

13. Odell S, Logan DE. Pediatric pain management: the multidisciplinary approach. J Pain Res (2013) 6: 785-90. doi: 10.2147/ JPR.S37434

14. Revivo G, Amstutz DK, Gagnon CM, et al. Interdisciplinary pain management improves pain and function in pediatric patients with chronic pain associated with joint hypermobility syndrome. PM R (2019) 11(2): 150-7. doi: 10.1016/j.pmrj.2018.06.018

15. Kozlowska K, Spooner CJ, Palmer DM, et al. 'Motoring in idle': the default mode and somatomotor networks are overactive in children and adolescents with functional neurological symptoms. Neuroimage Clin (2018) 18: 730-43. doi: 10.1016/j. nicl.2018.02.003

\section{* Marianna Kogut}

Kennedy Krieger Institute

Baltimore, MD, USA

Email: mckogut@gmail.com 